\title{
Review Article \\ Functional Performance Testing and Patient Reported Outcomes following ACL Reconstruction: A Systematic Scoping Review
}

\author{
Adel Almangoush and Lee Herrington \\ College of Health, Sport and Rehabilitation Sciences, University of Salford, Salford M6 6PU, UK \\ Correspondence should be addressed to Adel Almangoush; a.a.almangoush@edu.salford.ac.uk
}

Received 20 April 2014; Revised 11 September 2014; Accepted 22 October 2014; Published 17 November 2014

Academic Editor: Nadr Jomha

Copyright ( 2014 A. Almangoush and L. Herrington. This is an open access article distributed under the Creative Commons Attribution License, which permits unrestricted use, distribution, and reproduction in any medium, provided the original work is properly cited.

\begin{abstract}
Objective. A systematic scoping review of the literature to identify functional performance tests and patient reported outcomes for patients who undergo anterior cruciate ligament (ACL) reconstruction and rehabilitation that are used in clinical practice and research during the last decade. Methods. A literature search was conducted. Electronic databases used included Medline, PubMed, Cochrane Library, EMBASE, CINAHL, SPORTDiscus, PEDro, and AMED. The inclusion criteria were English language, publication between April 2004 and April 2014, and primary ACL reconstruction with objective and/or subjective outcomes used. Two authors screened the selected papers for title, abstract, and full-text in accordance with predefined inclusion and exclusion criteria. The methodological quality of all papers was assessed by a checklist of the Critical Appraisal Skills Programme (CASP). Results. A total of 16 papers were included with full-text. Different authors used different study designs for functional performance testing which led to different outcomes that could not be compared. All papers used a measurement for quantity of functional performance except one study which used both quantity and quality outcomes. Several functional performance tests and patient reported outcomes were identified in this review. Conclusion. No extensive research has been carried out over the past 10 years to measure the quality of functional performance testing and control stability of patients following ACL reconstruction. However this study found that the measurement of functional performance following ACL reconstruction consisting of a one-leg hop for a set distance or a combination of different hops using limb symmetry index (LSI) was a main outcome parameter of several studies. A more extensive series of tests is suggested to measure both the quantitative and qualitative aspects of functional performance after the ACL reconstruction. The KOOS and the IKDC questionnaires are both measures that are increasingly being used for ACL reconstruction throughout the last decade.
\end{abstract}

\section{Introduction}

Reconstruction surgery is very common to restore a rupture of an anterior cruciate ligament (ACL). There is currently a multiplicity of functional performance tests and patient reported outcome measures to determine the success of this surgery and rehabilitation [1-4]. For instance, the review done by Garratt et al. found more than 15 patient-assessed health instruments specific to the knee in the 31 studies that were included [5]. Also, Wang et al. identified twenty-four unique instrument outcomes measurements for the knee [6]. Regarding functional performance tests the review done by Clark reported that more than 18 tests were used to evaluate the function of lower extremity following an ACL deficiency or ACL reconstruction [7]. In light of the abundance of tests available, there appears no consensus regarding which test or combination of tests is most appropriate for evaluating recovery following ACL reconstruction [2]. It has been recommended that a multiplicity of assessments, incorporating both functional performance testing and patient reported tools, is important to evaluate functional ability and outcome for patients following ACL reconstruction [2], but which of these tests or combination of tests provides the most rigorous test for outcome remains unclear. As no single instrument or functional performance test is currently capable of measuring all the multitude of parameters believed to relate to outcome, 
it is rational to accept that a range of tests should be administered to facilitate a full comprehensive evaluation of outcome.

Functional performance testing is likely to indicate the outcome of the neuromuscular training and appears to consist of two components [8]. The first component is the quantity of movement or the capabilities of the production of the force, for example, muscle strength measurements and hop tests [8]. The second component is the quality of movement, for example, the total knee flexion when landing from a jump or the occurrence of dynamic knee valgus [9, 10]. These two components are important in rehabilitation and prevention of ACL recurrent injuries or surgery failure $[8,11,12]$. Most papers describing the functional performance following ACL reconstruction are using the limb symmetry index (LSI) and thus are limited to quantitative measurements $[13,14]$. Functional performance testing using qualitative methods evaluates compensation, or asymmetry, through clinical observation [15].

The limb symmetry index (LSI) calculation is commonly used when reporting the results of functional hop tests. The LSI is the percentage deficit of the distance hopped on the involved leg compared with the contralateral noninvolved leg [7]. The use of the LSI minimizes the probable confounding variable of the biological variation between people, which may affects the results [16]. The work of Munro and Herrington [17] showed LSI needs to be in excess of $90 \%$ to be deemed normal.

A functional outcome is a predicted result of care that is meaningful and practical for the patients and sustainable beyond the rehabilitation environment [18]. Functional outcomes not only assess benefits but also provide cost-benefit data. There are advantages and limitations to each measure used independently or in conjunction with other measures [18]. The practicality of functional outcome measures employed in the clinical/research setting is an important consideration [18]. Functional or performance tests provide an objective assessment of components of the patients' ability in a structured, controlled setting. Combining several tests to assess function may serve to minimize any trade-offs between specificity and sensitivity [19].

Regardless of which tests are selected, it is imperative that they be standardised, reliable, valid, and responsive to change with time as well as being clinically relevant [20-22]. Ideally, outcome measures in research and clinical practice should be low-cost, take an acceptable length of time to administer, be convenient for researcher and clinicians to use, and be acceptable to the participants under investigation [21, 22]. Therefore, the purpose of this scoping review was to identify and explore a number of commonly used outcome measures for patients following ACL reconstruction and postoperative rehabilitation to assess both aspects (quantitative and qualitative) of functional performance tests and self-reported questionnaires that have been used in last decade.

\section{Methodology}

We adopted a "systematic" scoping review approach-this is a combination of a scoping review methodology—to ensure the inclusion of broad areas of research and study designs and a systematic review of the methodology of the reviews [39]. A scoping review is a relatively new type of study providing an assessment of available evidence from the literature in a broad area of research such as the compliance in the reporting of clinical studies to established guidelines. It also serves to identify information gaps in the field and provide recommendations for implementation [39].

The methodology of scoping reviews was first described in detail by Arksey and O'Malley [39] in their pivotal paper published in 2005, which provided the foundation for carrying out a scoping review. This framework was further refined, and five stages were proposed to be followed when conducting a scoping review, including (1) the identification of a research question; (2) finding the relevant studies; (3) the selection of studies to be included in the review; (4) data extraction from the included studies; and (5) assembling, summarizing, and reporting the results of the review [40].

2.1. Search Strategy. A PRISMA compliant search strategy was used for study selection. The inclusion criteria of studies were as follows: (1) at least one lower extremity/knee functional performance test used as an outcome measurement of the article and/or patient reported outcomes, (2) subjects who were post-ACL reconstruction, (3) studies which were either randomised control trial (RCT), cross-sectional, or cohort designs, and (4) studies published in English between April 2004 and April 2014.

The electronic databases used were MEDLINE (MeSH terms), PubMed, Cochrane Library (systematic reviews and controlled trials registers), EMBASE, CINAHL, SPORTDiscus, PEDro (Physiotherapy Evidence database), and AMED (Allied and Complementary Medicine Index). In order to capture as many relevant references as possible, an expanded search was performed, including hand-searching the reference lists of all relevant articles, texts, and systematic reviews.

Search was conducted using the terms "knee" AND "ACL injuries" OR "functional performance" AND "measure" OR "test” OR "screen" OR “assessment" Or "patient reported." The keyword search was also performed on PubMed utilising the key terms "anterior cruciate ligament" AND "surgery" AND "injury" AND "physical performance outcome measurements" to ensure a detailed and comprehensive search strategy, and the additional search was performed in academic textbook that contained an extensive review of functional performance tests [41] (see Table 1).

2.2. Study Identification. Two reviewers (Adel Almangoush and Lee Herrington) independently reviewed all titles and abstracts that were identified from the search strategy. In accordance with the predefined eligibility criteria the full-text manuscripts for all potentially eligible studies were obtained, and then in accordance with the predefined eligibility criteria the reviewers independently reviewed them a second time.

2.3. Data Extraction. Data extraction for each eligible paper was performed independently by two reviewers (Adel Almangoush and Lee Herrington) using a predefined spreadsheet. The reviewers' spreadsheets were amalgamated to 
TABLE 1: Search terms adopted for a Medline search strategy.

\begin{tabular}{lc}
\hline Number & Search term \\
\hline 1 & Functional \\
2 & Performance \\
3 & Measure \\
4 & Screen \\
5 & Assessment \\
6 & Objective \\
7 & Subjective \\
8 & Questionnaire \\
9 & Surgery \\
10 & ACL \\
11 & Knee \\
12 & Injury \\
13 & Anterior cruciate ligament \\
14 & OR/6-8 \\
15 & OR/10-12 \\
16 & OR/9-10 \\
17 & OR/1-2-3 \\
18 & OR/1-4 \\
19 & OR/5-7 \\
\hline
\end{tabular}

create an agreed extraction form. The standardised data extraction form included details on (a) focus of study, study design, participant details, outcome measure (functional performance tests and patient reported outcomes), and results. In cases where insufficient data were provided within the publication, attempts were made to contact all corresponding authors to identify such data.

2.4. Critical Appraisal. Each study's methodological quality was assessed by using an appraisal tool devised to specifically evaluate functional performance testing and patients' reported questionnaires of studies that included those patients following ACL reconstruction. This was based on the Critical Appraisal Skills Programme (CASP) critical appraisal tool (CASP, 2007) [42], which has been widely used and employed in previous systematic reviews to evaluate the methodological quality of clinical studies [43-45]. The tool assessed domains such as the identification of the research questions, appropriateness of the research design, surgery and rehabilitation outcomes, the accuracy of description of methodology and population, appropriateness of analysis methods, and interpretation of findings. The appraisal was independently undertaken by two aforementioned reviewers (Adel Almangoush and Lee Herrington). If any disagreements arose regarding the study selection, data extraction, or appraisal score, these were sorted out through discussion between the two reviewers until a consensus was met. Studies were excluded if they achieved a very low methodological score of less than $50 \%$ through the CASP scoring system. A total score was calculated by adding up all positive items.

2.5. Data Analysis. All analyses were initially undertaken by one reviewer (Adel Almangoush) and verified by the other reviewer (Lee Herrington). A narrative review was undertaken of all included literature. An assessment of the quantity and quality of functional performance testing and patient reported tools of those patients following ACL reconstruction by means of a meta-analysis was planned. However, unfortunately due to the heterogeneity of the studies, in particular the information regarding surgery and rehabilitation outcomes, it was not possible to complete this analysis.

\section{Results}

3.1. Search Strategy. A PRISMA compliant search strategy was used, and results are presented in a PRISMA flow diagram (Figure 1) [46]. As Figure 1 demonstrates, a total of 196 citations were identified through the search strategy. Sixteen papers satisfied the eligibility criteria and were therefore included in the review. This included 10 randomised controlled trials and 6 cohort studies. These were summarised in Table 2.

3.2. Knee Laxity. Eleven studies assessed knee laxity using a variety of instrumented laxity tests. Nine studies used a KT1000 arthrometer (MEDmetric, San Diego, CA, USA) [26, 27, 29, 31, 33-37]. One study used a manual maximum test with a Rolimeter (Aircast, Summit, NJ, USA) [32]. One study used Lachman test and/or pivot shift test [30]. All studies assessed the anterior displacement of the tibia relative to the femur, except one study which used medial joint space opening on manual valgus stress testing [32].

3.2.1. Critical Appraisal. The findings of the critical appraisal are summarised in Table 3. On analysis, the literature presented with a number of methodological limitations. Only six papers (38\%) justified their sample sizes based on power calculations. Whilst the surgery management strategies undertaken were clearly described in most of these papers, only four publications presented sufficient information to reproduce their methodologies for physiotherapy treatments and described the rehabilitation programs undertaken (25\%). Furthermore, whilst all studies reviewed used appropriate outcome measures to evaluate their participants, only a few of them defined the presence of an observer. Whilst inferential statistics were presented in all included publications, confidence intervals were only provided in four papers (25\%). No study presented a standard error of measurement. None of the included studies evaluated the patients before the ACL operation. However, all authors interpreted their findings appropriately and related these results in a suitable manner to clinical practice and the existing evidence base. All papers passed more than $50 \%$.

\subsubsection{Study Description}

Outcome Measures. A variety of different functional performance tests and patient reported outcomes measures have been reported in patients following ACL reconstruction. These were assessed individually as shown below. 


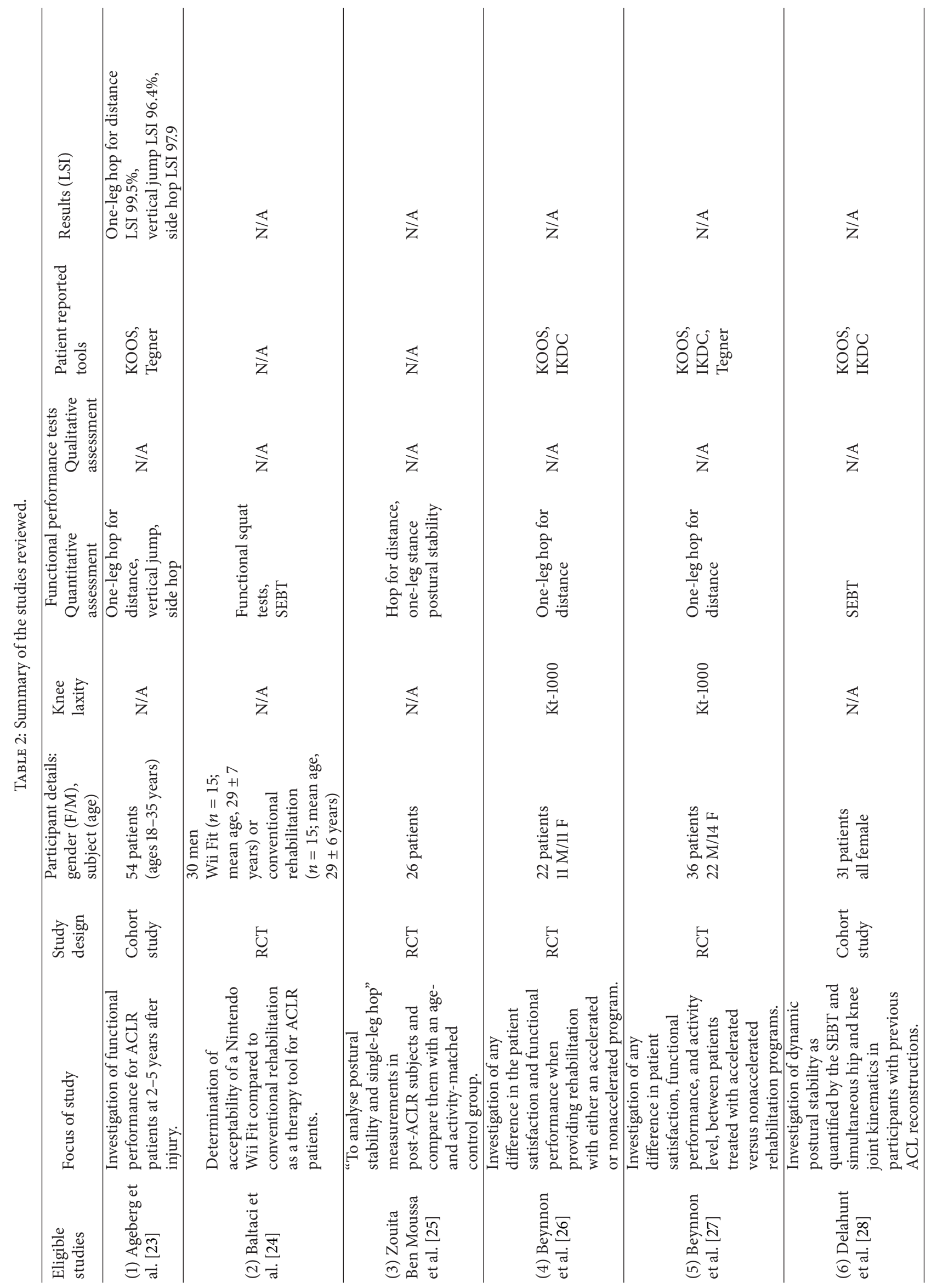




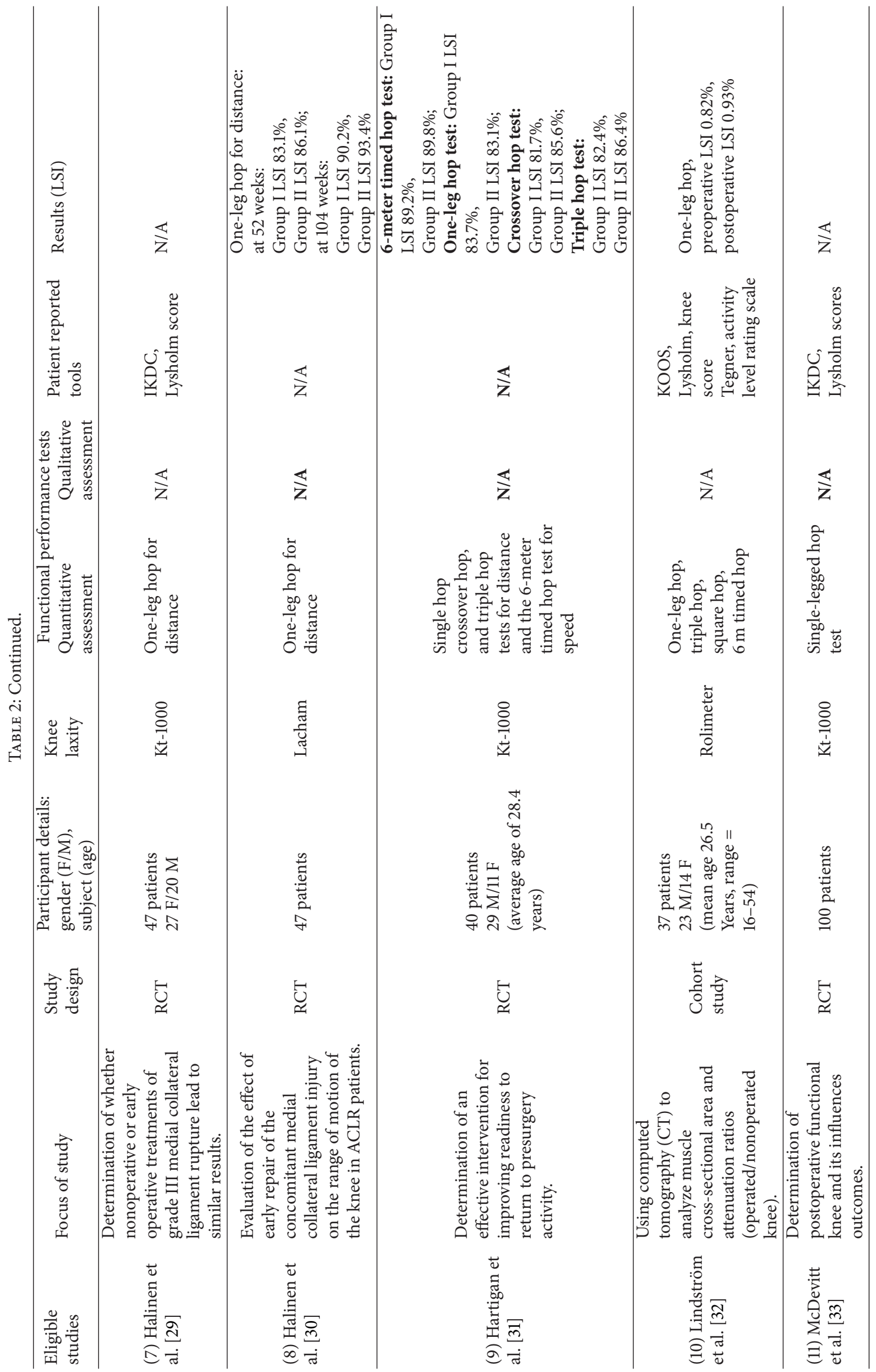




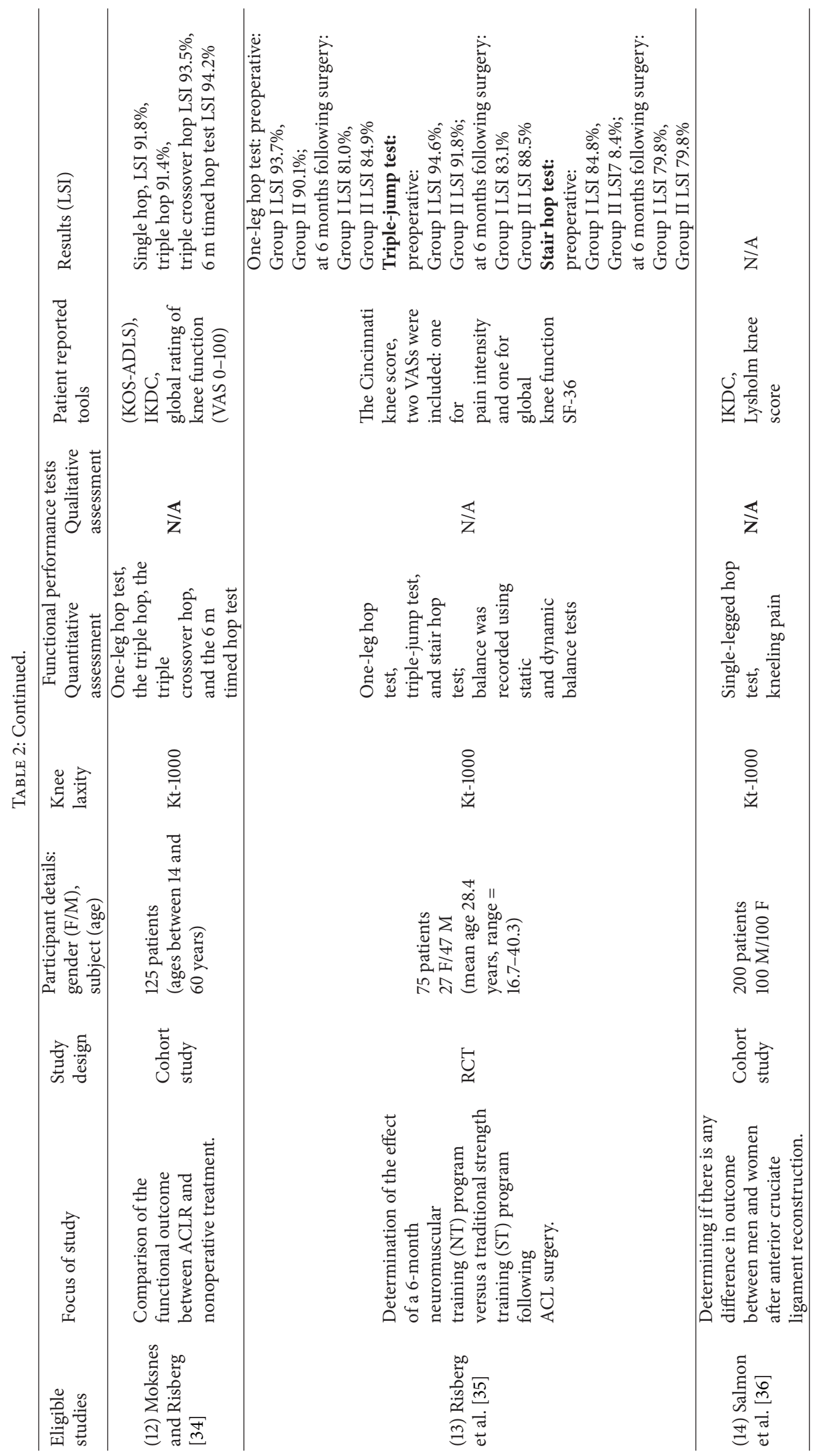




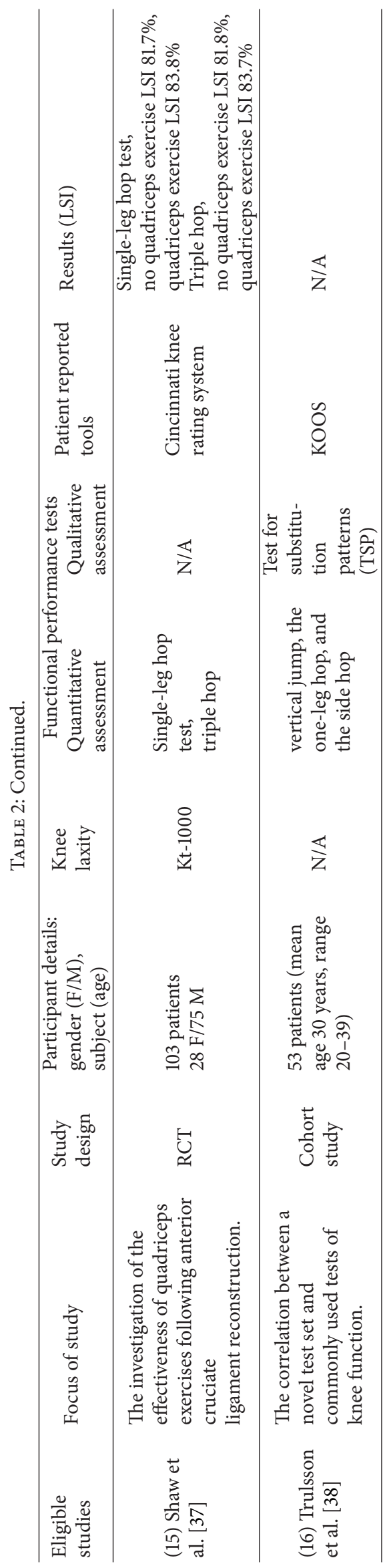




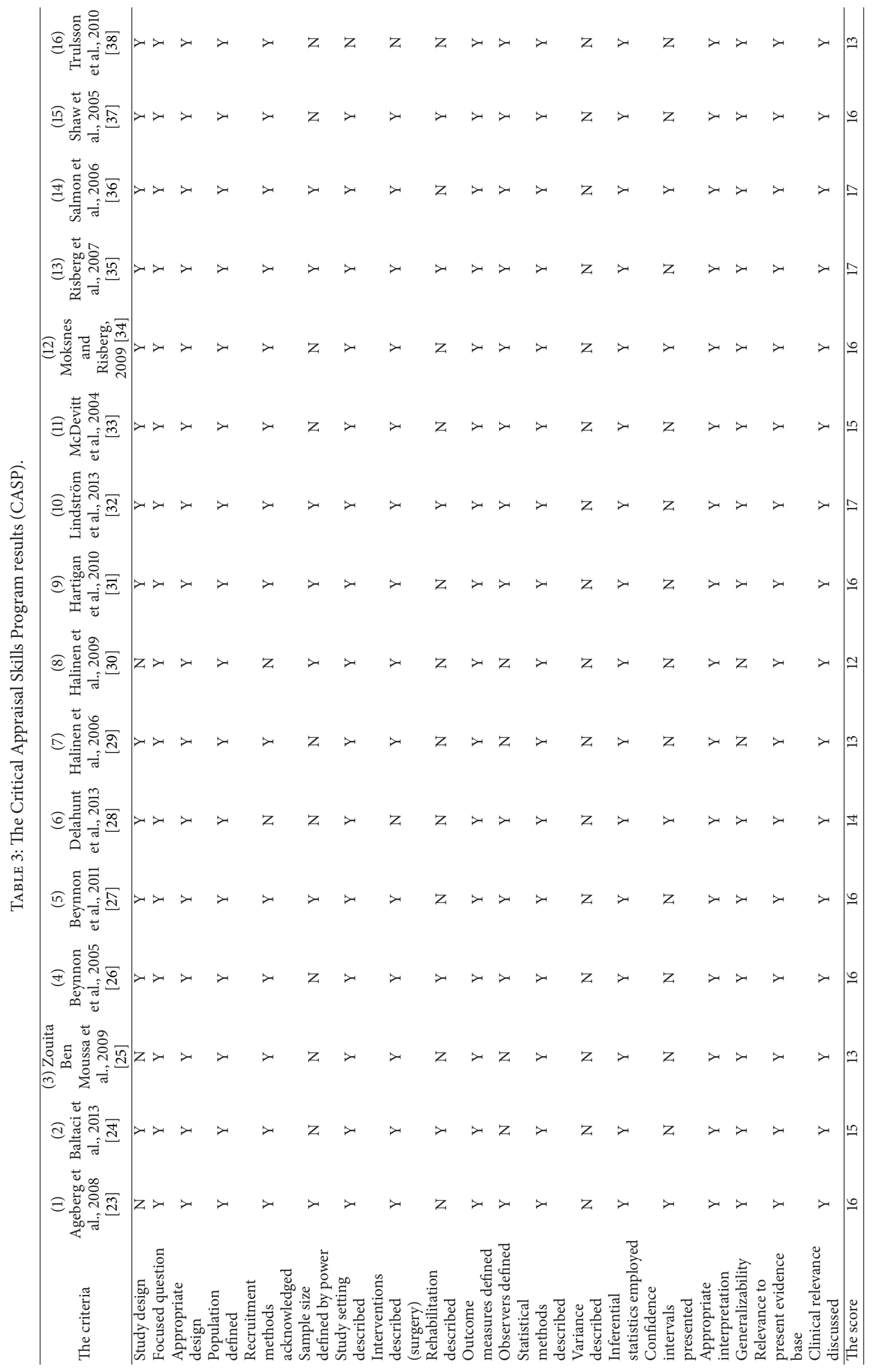




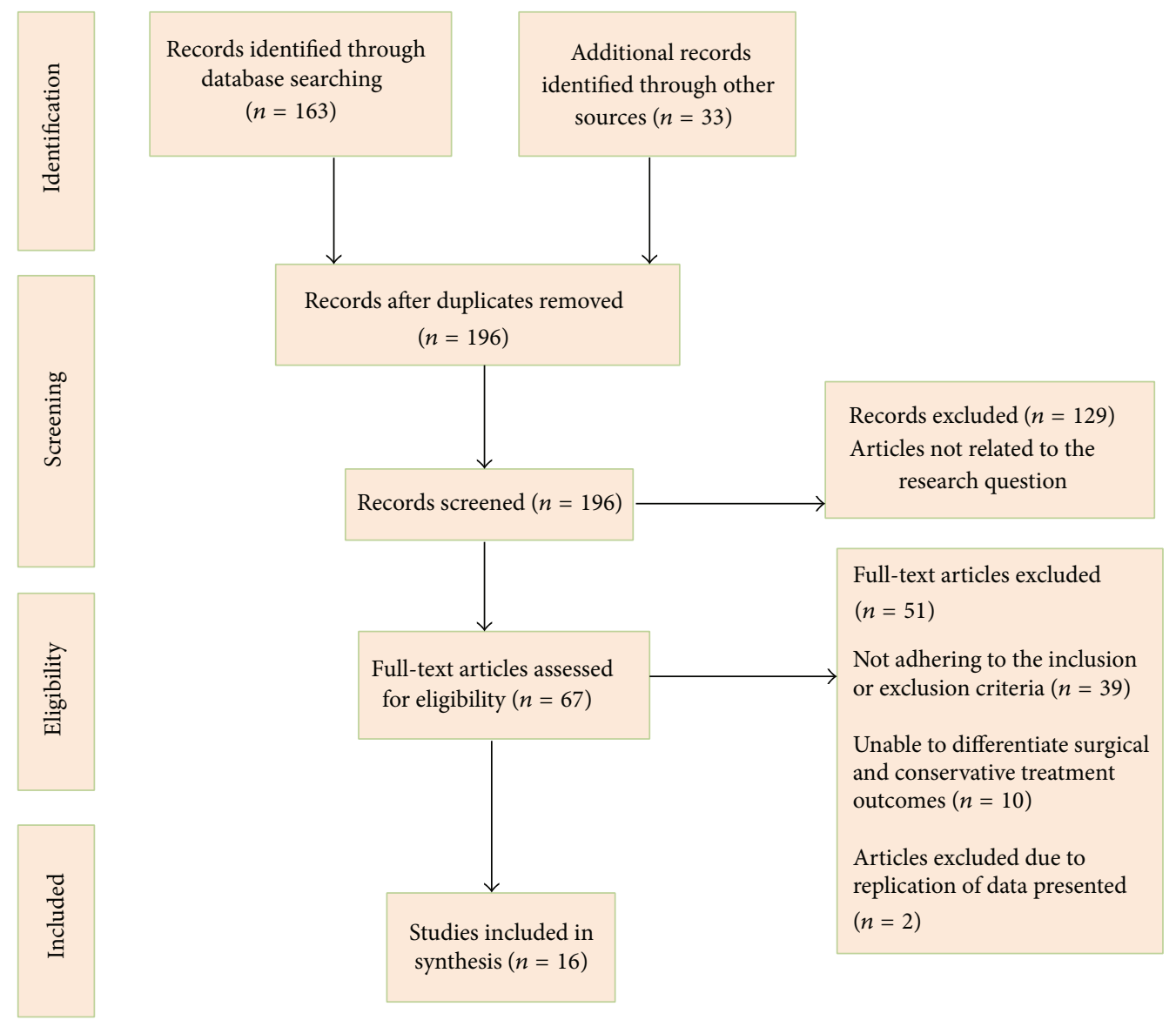

FiguRE 1: PRISMA follow diagram to depict search strategy results.

\subsection{Functional Performance Testing}

3.3.1. Hop Tests. A number of different assessment methods were used to determine the functional performance of patients following ACL reconstruction. These methods included the one-leg hope for distance; this is a commonly used functional performance test of both strength and confidence in the tested leg; it correlates positively with muscle strength and power [7, 47]. The one-leg hope for distance was assessed in fourteen studies (88\%) of the papers included. Triple hop test for distance was evaluated in four papers $[31,32,34,37]$. Three studies described a 6-meter timed hop test for speed [31, 32, 34]; crossover hop of distance was assessed in two studies $[31,34]$; side hop and vertical jump were also assessed in two studies [21, 38]; triple-jump test and stair hop test were evaluated in one study only [35]; and functional squat test was assessed in only one study also [24]. More than $50 \%$ of studies used the hop tests as a measurement of function within the battery of different tests completed. Only seven studies used multiple hop tests (44\%), and only seven papers (less than 50\%) reported limb symmetry index (LSI) comparing the injured with uninjured leg. Only one study described the quality of movement whilst carrying out the test (e.g., dynamic knee valgus or knee flexion angle) [38].
3.4. Postural Control. Postural stability of patients following ACL reconstruction was assessed in four studies by using different measurement methods. Baltaci et al. and Delahunt et al. used the modified star excursion balance test (SEBT) to evaluate the postural control of their patients $[24,28]$. Risberg et al. [35] and Zouita Ben Moussa et al. [25] used the NeuroCom Balance Master platform system to measure the postural stability. Balance was recorded using static and dynamic balance tests on an instrumented unstable platform (KAT2000).

3.5. Patient Reported Outcomes. Several reported questionnaires presented in the papers were evaluated in this scoping review, whereas KOOS and IKDC were assessed in most of the selected papers. Only four studies used Lysholm score $[29,32,33,36]$, three papers assessed the Tegner activity level rating scale $[23,27,32]$, and only two studies per each score evaluated the global rating scale $[31,34]$, the KOS-ADLS questionnaire [31, 34], and the Cincinnati knee score [35, 37].

Kocher et al. [48] made a comprehensive analysis of determinants of patient reported outcomes after ACL reconstruction. They concluded that subjective variables are more important for evaluation of patient reported outcomes than objective findings. They found 7 "key" symptoms that 
together accounted for $83 \%$ of the variability in patients reported outcomes.

\section{Discussion}

The authors of the current review aimed to identify existing functional performance testing and patient reported outcomes for patients following ACL reconstruction in the last decade. The most important finding of the present study was that all included articles used limited quantitative measurements to determine functional performance, except the study done by Trulsson et al. [38]. In the last decade most of the studies included in this review were focusing on the hop tests especially the single-leg hop test and few of these studies looked at a postural stability. Regarding the reported outcomes the focus was on the KOOS and IKDC questionnaires.

4.1. Functional Performance Testing. Although the included articles reported the use of several hop tests, fourteen studies used a single-leg hop for distance as the gold standard for measuring functional performance after ACL reconstruction because the reliability of this test is high (ICC ranging from 0.86 to 0.95$)[49,50]$. The relative reliability of the single hop for distance test in patients 1 to 2 years following ACL reconstruction has previously been reported [51]. However, several studies showed that the sensitivity increases when two or more different hop tests are performed [50-52]. By using multiple hop tests, their qualities can be assessed and thereby the opportunity to detect discrepancies in hop performance increases [49]. There is a strong relationship between crossover hop performance and functional outcome [38] correlating significantly to IKDC subjective and KOOS questionnaire scores [53]. The most reliable and valid of the multitude of hop tests in relation to the ACLR patient would appear to be the single hop for distance and the crossover hop tests $[7,50,54]$. The ability of the ACLR patient to perform well during hop tests is of paramount importance when judging functional performance.

Hop testing has frequently been proposed as a practical performance-based outcome measure that reflects the integrated effect of neuromuscular control, strength (forcegenerating capacity), and confidence in the limb and requires minimal equipment and time to administer [55]. Based on a review of the potential use of hop tests as measures of dynamic knee stability, Fitzgerald et al. [56] suggested that hopping may be appropriate for use as a predictive tool for identifying patients who may have future problems as a result of knee injury or pathology and as an evaluative tool to reflect change in the patient status in response to treatment.

Within the published literature, the "gold standard" is often regarded as having a limb symmetry index (LSI) of greater than $85 \%$ [7], indicating that anything less than a $15 \%$ deficit in strength between the operated and nonoperated limb is acceptable. This works on the assumption that the uninjured limb is "normal" in terms of its strength [7]. A study conducted in [57] has shown that the contralateral (noninjured) leg is significantly weaker than matched controls. Therefore, this assumption of normality should be viewed with caution, as the period of time during both preoperative and postoperative rehabilitation is likely to have caused atrophy of the noninjured leg. However, using the LSI is debatable because recent studies have shown that an ACL injury could lead to a crossover effect in the uninvolved leg resulting in strength and function loss based on biomechanical and neuromuscular changes [16].

4.2. Postural Control. To the best of the knowledge of this study's researchers, there are few published studies that search for postural stability following ACL reconstruction [58]. For example, the SEBT outcome measure offers a simple, reliable, valid, and low-cost alternative to more sophisticated instrumented methods, to assess dynamic balance ability [59, 60]; unlike force plates or electronically controlled balance platforms, it is an easy and highly portable test that could be employed in a range of clinical environments. According to Logerstedt et al. [54] the grid required testing for ACL deficiency patients, three lines are positioned on the grid (anterior, medial, and lateral reach distance) which are labelled according to the direction of excursion relative to the stance leg.

High intertester reliability of the SEBT has previously been reported [59]. Whilst previous studies have evaluated intratester reliability [59], only one study has evaluated between-session reliability of the SEBT with normalised scores with ICC values ranging from 0.89 to 0.93 [61]. However, only 3 reach distances, anterior, posteromedial, and posterolateral, were evaluated. Therefore, further study of between-session reliability of all reach directions is warranted.

Previous research has suggested that the SEBT is reliable and sensitive enough to detect dynamic postural control deficits in patients with an ACL-deficient (ACL-D) limb $[62,63]$. In these studies, patients who were injured were shown to have lower SEBT scores compared to those of their uninjured limb and those of healthy participants. In particular, Herrington et al. [62] found that patients with ACL deficiency showed functional deficits in the anterior, medial, lateral, and posteromedial reach directions.

Functional tests are a quick and inexpensive method of obtaining an objective measure of lower limb function following surgery [52]. These tests are thought to provide an indication of muscle strength and power, neuromuscular control and confidence $[64,65]$. Additionally, a number of authors have highlighted that a single functional test may not be sensitive enough to detect performance limitations and that at least two functional tests should be used $[51,52,55]$.

Furthermore, all included studies reported quantitative data such as distance and/or time. Only one study described the quality of movement whilst carrying out the test (e.g., dynamic knee valgus or knee flexion angle) [38]. Studies focusing on prevention showed that the risk for ACL injuries was reduced when training was done before high quality trials [66-68]. For ACL injury screening, Ekegren and his colleagues examined dynamic knee valgus during a dropjump task. The drop jump turned out to be a reliable and valid instrument in observing the dynamic knee valgus 
[9]. von Porat et al. investigated videotaped functional performance tests in ACL injured subjects, and they reported that observation is a reliable and valid instrument for assessing knee flexion angles of the one-leg hop for a distance [10]. The single-leg squat (SLS) test is a cost-effective and simple movement to determine lower extremity alignment in the coronal plane. Carried out with a single camera in any setting, this procedure can visibly identify a valgus lower extremity alignment on landing, which is considered to be a potential risk factor for a possible noncontact ACL injury [12]. The SLS test has been described in a number of studies as a useful clinical measure to identify hip muscle function and dynamic knee control [69].

4.3. Patient Reported Outcomes. Patient reported instruments are normally related to signs and symptoms experienced by the patient and/or the functional tasks that individuals are able to achieve during their activities of daily living [65]. A commonly used knee outcome instrument is the Cincinnati knee scoring scale, and although it has been demonstrated to be an adequate tool to evaluate knee function following ACL reconstruction [70], it also includes manual and instrumented stability testing to assess symptoms and function; thus it becomes more difficult to separate various aspects of knee function following ACL injury.

The International Knee Documentation Committee (IKDC) developed a scoring system for knees with ACL injuries. The IKDC is reliable and the validity and responsiveness were found to be good [71]. The IKDC, the Cincinnati knee scoring scale, and the first version of the Lysholm score are assessor reported scores, which have been exposed to be biased when applied to individuals with an ACL injury [72]. On the other hand, the Lysholm-Tegner system is much simpler but mainly evaluates symptoms and activity. Carlos argued that for those clinicians and researchers considering using only the IKDC as their patient reported outcomes for ACL reconstruction, they should include as a minimum the KOOS subscales that address broader areas of concern, including quality of life and emotional health that are most important to patients following ACL reconstruction and are not wholly represented in IKDC [73]. Moreover, there is a suggestion that the KOOS is perhaps more suitable for the assessment of patients in the longer term unlike the IKDC [74]. The KOOS has shown good validity and demonstrated that it is responsive to ACL reconstruction and rehabilitation; it shows that it is a reliable instrument for patients undergoing ACL surgery and rehabilitation [74]. KOOS has been used in an extensive amount of current research protocols and it has been translated and culturally adapted into various languages [75]. Clinicians and researchers looking to use a patient-based score measure of outcomes must consider the specific patient population in which it has been evaluated. Using a diagnostic algorithm that measures the anatomic parts of the knee as separate constructs may solve this dilemma, allowing for the measurement of treatment outcomes across patient groups and the selection of the optimal clinical intervention.

In general, the papers in this literature review included poorly described sample sizes and whether or not the sample size was based on power calculations. Accordingly, the samples recruited may not necessarily have been big enough to identify a difference in outcome following a rehabilitation programme, irrespective of whether or not a difference existed [76]. The papers weakly described who had assessed the subjects. Accordingly, it was not possible to determine whether measurement error influenced the results obtained or whether the experiences or training of the assessors was a variable which may have accounted for any between-study differences.

Limitations. There are limitations of this systematic scoping review that should be acknowledged. For instance, the authors established very specific inclusion/exclusion criteria for selection of functional performance tests included inthis review. This included only the functional performance tests for ACL reconstruction patients after surgery. Many tests were excluded because the studies were performed on healthy people or subjects with various neurological or debilitating comorbidities. Therefore, it is possible that some functional performance tests were not identified. This may modify the interpretation of the values attained for a specific functional performance test, and this was also the reason for the small number of studies included.

Future studies are required to establish the reliability and validity of existing functional performance tests or explore new, relevant quality measurements of the functional performance tests to be used in patients following ACL surgery.

\section{Conclusion}

This review shows that, following the ACL reconstruction, the one-leg hop for distance or a combination of different hops and the limb symmetry index (LSI) of functional performance tests was used as a main outcome parameter of several studies. No extensive research has been carried out over the past 10 years to measure the control stability of patients following ACL reconstruction. Furthermore, no observation or videotaping was used to assess the quality of any test of any functional performance and control stability of ACL patients following surgery except for one study. Because previous studies discuss additional important parameters, a more extensive battery of tests is suggested to measure both the quantitative and qualitative aspects of functional performance after the ACL reconstruction. The KOOS and the IKDC are both measures that are increasingly being used for ACL reconstruction during the last 10 years.

\section{Conflict of Interests}

The authors declare that there is no conflict of interests regarding the publication of this paper.

\section{References}

[1] E. Linko, A. Harilainen, A. Malmivaara, and S. Seitsalo, "Operative treatment for anterior cruciate ligament ruptures in adults 
(Protocol for Cochrane Review)," The Cochrane Review 2, Update Software, Oxford, UK, 2000.

[2] N. Phillips, M. Benjamin, T. Everett, and R. W. M. van Deursen, "Outcome and progression measures in rehabilitation following anterior cruciate ligament injury," Physical Therapy in Sport, vol. 1, no. 4, pp. 106-118, 2000.

[3] Y. Tegner and J. Lysholm, "Rating systems in the evaluation of knee ligament injuries," Clinical Orthopaedics and Related Research, vol. 198, pp. 43-49, 1985.

[4] T. Shaw, L. S. Chipchase, and M. T. Williams, "A users guide to outcome measurement following ACL reconstruction," Physical Therapy in Sport, vol. 5, pp. 57-67, 2004.

[5] A. M. Garratt, S. Brealey, W. J. Gillespie, and DAMASK Trial Team, "Patient-assessed health instruments for the knee: a structured review," Rheumatology, vol. 43, no. 11, pp. 1414-1423, 2004.

[6] D. Wang, M. H. Jones, M. M. Khair, and A. Miniaci, "Patientreported outcome measures for the knee," The Journal of Knee Surgery, vol. 23, no. 3, pp. 137-151, 2010.

[7] N. C. Clark, "Functional performance testing following knee ligament injury," Physical Therapy in Sport, vol. 2, no. 2, pp. 91105, 2001.

[8] E. Ageberg, "Consequences of a ligament injury on neuromuscular function and relevance to rehabilitation-using the anterior cruciate ligament-injured knee as model," Journal of Electromyography \& Kinesiology, vol. 12, no. 3, pp. 205-212, 2002.

[9] C. L. Ekegren, W. C. Miller, R. G. Celebrin, J. J. Eng, and D. L. MacIntyre, "Reliability and validity of observational risk screening in evaluating dynamic knee valgus," Journal of Orthopaedic \& Sports Physical Therapy, vol. 39, no. 9, pp. 665674, 2009.

[10] A. von Porat, E. Holmstöm, and E. Roos, "Reliability and validity of videotaped functional performance tests in ACLinjured subjects," Physiotherapy Research International, vol. 13, no. 2, pp. 119-130, 2008.

[11] R. Thomeé, Y. Kaplan, J. Kvist et al., "Muscle strength and hop performance criteria prior to return to sports after ACL reconstruction," Knee Surgery, Sports Traumatology, Arthroscopy, vol. 19, no. 11, pp. 1798-1805, 2011.

[12] M. V. Paterno, L. C. Schmitt, K. R. Ford et al., "Biomechanical measures during landing and postural stability predict second anterior cruciate ligament injury after anterior cruciate ligament reconstruction and return to sport," American Journal of Sports Medicine, vol. 38, no. 10, pp. 1968-1978, 2010.

[13] G. B. Maletis, S. L. Cameron, J. J. Tengan, and R. J. Burchette, "A prospective randomized study of anterior cruciate ligament reconstruction: a comparison of patellar tendon and quadruple-strand semitendinosus/gracilis tendons fixed with bioabsorbable interference screws," The American Journal of Sports Medicine, vol. 35, no. 3, pp. 384-394, 2007.

[14] A. Heijne and S. Werner, "A 2-year follow-up of rehabilitation after ACL reconstruction using patellar tendon or hamstring tendon grafts: a prospective randomised outcome study," Knee Surgery, Sports Traumatology, Arthroscopy, vol. 18, no. 6, pp. 805-813, 2010.

[15] S. M. Lephart and T. J. Henry, "Functional rehabilitation for the upper and lower extremity," The Orthopedic Clinics of North America, vol. 26, no. 3, pp. 579-592, 1995.

[16] P. Swärd, I. Kostogiannis, and H. Roos, "Risk factors for a contralateral anterior cruciate ligament injury," Knee Surgery,
Sports Traumatology, Arthroscopy, vol. 18, no. 3, pp. 277-291, 2010.

[17] A. G. Munro and L. C. Herrington, "Between-session reliability of four hop tests and the agility T-test," The Journal of Strength \& Conditioning Research, vol. 25, no. 5, pp. 1470-1477, 2011.

[18] D. R. Keskula, J. B. Duncan, V. L. Davis, and P. W. Finley, "Functional outcome measures for knee dysfunction assessment," Journal of Athletic Training, vol. 31, no. 2, p. 105, 1996.

[19] L. G. Portney and M. P. Watkins, Foundations of Clinical Research: Applications to Practice, Prentice Hall, Upper Saddle River, NJ, USA, 2008.

[20] D. S. Johnson and R. B. Smith, "Outcome measurement in the ACL deficient knee-what's the score?" Knee, vol. 8, no. 1, pp. 51-57, 2001.

[21] R. Hammond, "Evaluation of physiotherapy by measuring the outcome," Physiotherapy, vol. 86, no. 4, pp. 170-172, 2000.

[22] M. Law, "Measurement in occupational therapy: scientific criteria for evaluation," Canadian Journal of Occupational Therapy, vol. 54, no. 3, pp. 133-138, 1987.

[23] E. Ageberg, R. Thomeé, C. Neeter, K. G. Silbernagel, and E. M. Roos, "Muscle strength and functional performance in patients with anterior cruciate ligament injury treated with training and surgical reconstruction or training only: a two to five-year followup," Arthritis Care and Research, vol. 59, no. 12, pp. 17731779, 2008.

[24] G. Baltaci, G. Harput, B. Haksever, B. Ulusoy, and H. Ozer, "Comparison between Nintendo Wii Fit and conventional rehabilitation on functional performance outcomes after hamstring anterior cruciate ligament reconstruction: prospective, randomized, controlled, double-blind clinical trial," Knee Surgery, Sports Traumatology, Arthroscopy, vol. 21, no. 4, pp. 880-887, 2013.

[25] A. Zouita Ben Moussa, S. Zouita, C. Dziri, and F. Z. Ben Salah, "Single-leg assessment of postural stability and knee functional outcome two years after anterior cruciate ligament reconstruction," Annals of Physical and Rehabilitation Medicine, vol. 52, no. 6, pp. 475-484, 2009.

[26] B. D. Beynnon, B. S. Uh, R. J. Johnson et al., "Rehabilitation after anterior cruciate ligament reconstruction: a prospective, randomized, double-blind comparison of programs administered over 2 different time intervals," The American Journal of Sports Medicine, vol. 33, no. 3, pp. 347-359, 2005.

[27] B. D. Beynnon, R. J. Johnson, S. Naud et al., "Accelerated versus nonaccelerated rehabilitation after anterior cruciate ligament reconstruction: a prospective, randomized, doubleblind investigation evaluating knee joint laxity using roentgen stereophotogrammetric analysis," The American Journal of Sports Medicine, vol. 39, no. 12, pp. 2536-2548, 2011.

[28] E. Delahunt, M. Chawke, J. Kelleher et al., "Lower limb kinematics and dynamic postural stability in anterior cruciate ligamentreconstructed female athletes," Journal of Athletic Training, vol. 48, no. 2, pp. 172-185, 2013.

[29] J. Halinen, J. Lindahl, E. Hirvensalo, and S. Santavirta, "Operative and nonoperative treatments of medial collateral ligament rupture with early anterior cruciate ligament reconstruction: a prospective randomized study," The American Journal of Sports Medicine, vol. 34, no. 7, pp. 1134-1140, 2006.

[30] J. Halinen, J. Lindahl, and E. Hirvensalo, "Range of motion and quadriceps muscle power after early surgical treatment of acute combined anterior cruciate and grade-III medial collateral ligament injuries: a prospective randomized study," The Journal of Bone and Joint Surgery, vol. 91, no. 6, pp. 1305-1312, 2009. 
[31] E. H. Hartigan, M. J. Axe, and L. Snyder-Mackler, "Time line for noncopers to pass return-to-sports criteria after anterior cruciate ligament reconstruction," Journal of Orthopaedic and Sports Physical Therapy, vol. 40, no. 3, pp. 141-154, 2010.

[32] M. Lindström, S. Strandberg, T. Wredmark, L. Felländer-Tsai, and M. Henriksson, "Functional and muscle morphometric effects of ACL reconstruction. A prospective CT study with lyear follow-up," Scandinavian Journal of Medicine and Science in Sports, vol. 23, no. 4, pp. 431-442, 2013.

[33] E. R. McDevitt, D. C. Taylor, M. D. Miller et al., "Functional bracing after anterior cruciate ligament reconstruction: a prospective, randomized, multicenter study," The American Journal of Sports Medicine, vol. 32, no. 8, pp. 1887-1892, 2004.

[34] H. Moksnes and M. A. Risberg, "Performance-based functional evaluation of non-operative and operative treatment after anterior cruciate ligament injury," Scandinavian Journal of Medicine and Science in Sports, vol. 19, no. 3, pp. 345-355, 2009.

[35] M. A. Risberg, I. Holm, G. Myklebust, and L. Engebretsen, "Neuromuscular training versus strength training during first 6 months after anterior cruciate ligament reconstruction: a randomized clinical trial," Physical Therapy, vol. 87, no. 6, pp. 737-750, 2007.

[36] L. J. Salmon, K. M. Refshauge, V. J. Russell, J. P. Roe, J. Linklater, and L. A. Pinczewski, "Gender differences in outcome after anterior cruciate ligament reconstruction with hamstring tendon autograft," American Journal of Sports Medicine, vol. 34, no. 4, pp. 621-629, 2006.

[37] T. Shaw, M. T. Williams, and L. S. Chipchase, "Do early quadriceps exercises affect the outcome of ACL reconstruction? A randomised controlled trial," Australian Journal of Physiotherapy, vol. 51, no. 1, pp. 9-17, 2005.

[38] A. Trulsson, E. M. Roos, E. Ageberg, and M. Garwicz, "Relationships between postural orientation and self reported function, hop performance and muscle power in subjects with anterior cruciate ligament injury," BMC Musculoskeletal Disorders, vol. 11, no. 1, article 143, 2010.

[39] H. Arksey and L. O’Malley, "Scoping studies: towards a methodological framework," International Journal of Social Research Methodology, vol. 8, no. 1, pp. 19-32, 2005.

[40] S. E. Brien, D. L. Lorenzetti, S. Lewis, J. Kennedy, and W. A. Ghali, "Overview of a formal scoping review on health system report cards," Implementation Science, vol. 5, no. 1, article 2, 2010.

[41] M. P. Reiman and R. C. Manske, Functional Testing in Human Performance, Human Kinetics, Champaign, Ill, USA, 2009.

[42] CASP (Critical Skills Appraisal Programme), Learning \& Development Public Health Resource Unit, Oxford, UK, 2007, http://www.phru.nhs.uk/casp/critical.appraisal-tools.htm.

[43] K. A. Reilly, K. L. Barker, and D. Shamley, "A systematic review of lateral wedge orthotics-how useful are they in the management of medial compartment osteoarthritis?" Knee, vol. 13, no. 3, pp. 177-183, 2006.

[44] T. O. Smith, J. Walker, and N. Russell, "Outcomes of medial patellofemoral ligament reconstruction for patellar instability: a systematic review," Knee Surgery, Sports Traumatology, Arthroscopy, vol. 15, no. 11, pp. 1301-1314, 2007.

[45] T. O. Smith, L. Davies, and C. B. Hing, "A systematic review to determine the reliability of knee joint position sense assessment measures," Knee, vol. 20, no. 3, pp. 162-169, 2013.

[46] D. Moher, A. Liberati, J. Tetzlaff, and D. G. Altman, "Preferred reporting items for systematic reviews and meta-analyses: the PRISMA statement," BMJ, vol. 339, no. 7716, pp. 332-336, 2009.
[47] U. Wisløff, C. Castagna, J. Helgerud, R. Jones, and J. Hoff, "Strong correlation of maximal squat strength with sprint performance and vertical jump height in elite soccer players," British Journal of Sports Medicine, vol. 38, no. 3, pp. 285-288, 2004.

[48] M. S. Kocher, J. R. Steadman, K. Briggs, D. Zurakowski, W. I. Sterett, and R. J. Hawkins, "Determinants of patient satisfaction with outcome after anterior cruciate ligament reconstruction," The Journal of Bone and Joint Surgery, vol. 84, no. 9, pp. 1560$1572,2002$.

[49] A. Gustavsson, C. Neeter, P. Thomeé et al., "A test battery for evaluating hop performance in patients with an ACL injury and patients who have undergone ACL reconstruction," Knee Surgery, Sports Traumatology, Arthroscopy, vol. 14, no. 8, pp. 778-788, 2006.

[50] A. Reid, T. B. Birmingham, P. W. Stratford, G. K. Alcock, and J. R. Giffin, "Hop testing provides a reliable and valid outcome measure during rehabilitation after anterior cruciate ligament reconstruction," Physical Therapy, vol. 87, no. 3, pp. 337-349, 2007.

[51] D. M. Hopper, S. C. Goh, L. A. Wentworth et al., "Test-retest reliability of knee rating scales and functional hop tests one year following anterior cruciate ligament reconstruction," Physical Therapy in Sport, vol. 3, no. 1, pp. 10-18, 2002.

[52] S. D. Barber, F. R. Noyes, R. Mangine, and M. DeMaio, "Rehabilitation after ACL reconstruction: function testing," Orthopedics, vol. 15, no. 8, pp. 969-974, 1992.

[53] E. K. Reinke, K. P. Spindler, D. Lorring et al., "Hop tests correlate with IKDC and KOOS at minimum of 2 years after primary ACL reconstruction," Knee Surgery, Sports Traumatology, Arthroscopy, vol. 19, no. 11, pp. 1806-1816, 2011.

[54] D. Logerstedt, H. Grindem, A. Lynch et al., "Single-legged hop tests as predictors of self-reported knee function after anterior cruciate ligament reconstruction: the Delaware-Oslo ACL cohort study," The American Journal of Sports Medicine, vol. 40, no. 10, pp. 2348-2356, 2012.

[55] R. Petschnig, R. Baron, and M. Albrecht, "The relationship between isokinetic quadriceps strength test and hop tests for distance and one-legged vertical jump test following anterior cruciate ligament reconstruction," Journal of Orthopaedic \& Sports Physical Therapy, vol. 28, no. 1, pp. 23-31, 1998.

[56] G. K. Fitzgerald, S. M. Lephart, J. H. Hwang, and M. R. S. Wainner, "Hop tests as predictors of dynamic knee stability," The Journal of Orthopaedic and Sports Physical Therapy, vol. 31, no. 10, pp. 588-597, 2001.

[57] L. C. Schmitt, M. V. Paterno, and T. E. Hewett, "The impact of quadriceps femoris strength asymmetry on functional performance at return to sport following anterior cruciate ligament reconstruction," Journal of Orthopaedic and Sports Physical Therapy, vol. 42, no. 9, pp. 750-759, 2012.

[58] B. E. Howells, C. L. Ardern, and K. E. Webster, "Is postural control restored following anterior cruciate ligament reconstruction? A systematic review," Knee Surgery, Sports Traumatology, Arthroscopy, vol. 19, no. 7, pp. 1168-1177, 2011.

[59] J. Hertel, S. J. Miller, and C. R. Denegar, "Intratester and intertester reliability during the star excursion balance tests," Journal of Sport Rehabilitation, vol. 9, no. 2, pp. 104-116, 2000.

[60] L. C. Olmsted, C. R. Carciat, J. Hertel, and S. J. Shultz, "Efficacy of the star excursion balance tests in detecting reach deficits in subjects with chronic ankle instability," Journal of Athletic Training, vol. 37, no. 4, pp. 501-506, 2002. 
[61] P. J. Plisky, M. J. Rauh, T. W. Kaminski, and F. B. Underwood, "Star excursion balance test as a predictor of lower extremity injury in high school basketball players," Journal of Orthopaedic and Sports Physical Therapy, vol. 36, no. 12, pp. 911-919, 2006.

[62] L. Herrington, J. Hatcher, A. Hatcher, and M. McNicholas, "A comparison of Star Excursion Balance Test reach distances between ACL deficient patients and asymptomatic controls," Knee, vol. 16, no. 2, pp. 149-152, 2009.

[63] J. Hertel, R. A. Braham, S. A. Hale, and L. C. OlmstedKramer, "Simplifying the star excursion balance test: analyses of subjects with and without chronic ankle instability," Journal of Orthopaedic and Sports Physical Therapy, vol. 36, no. 3, pp. 131-137, 2006.

[64] W. D. Bandy, K. R. Rusche, and F. Y. Tekulve, "Reliability and limb symmetry for five unilateral functional tests of the lower extremities," Isokinetics and Exercise Science, vol. 4, no. 3, pp. 108-111, 1994.

[65] P. A. Borsa, S. M. Lephart, and J. J. Irrgang, "Comparison of performance-based and patient reported measures of function in anterior-cruciate-ligament-deficient individuals," Journal of Orthopaedic and Sports Physical Therapy, vol. 28, no. 6, pp. 392399, 1998.

[66] E. Alentorn-Geli, G. D. Myer, H. J. Silvers et al., "Prevention of non-contact anterior cruciate ligament injuries in soccer players. Part 2: a review of prevention programs aimed to modify risk factors and to reduce injury rates," Knee Surgery, Sports Traumatology, Arthroscopy, vol. 17, no. 8, pp. 859-879, 2009.

[67] T. E. Hewett, T. N. Lindenfeld, J. V. Riccobene, and F. R. Noyes, "The effect of neuromuscular training on the incidence of knee injury in female athletes: a prospective study," The American Journal of Sports Medicine, vol. 27, no. 6, pp. 699-706, 1999.

[68] W. Petersen, C. Braun, W. Bock et al., "A controlled prospective case control study of a prevention training program in female team handball players: the German experience," Archives of orthopaedic and trauma surgery, vol. 125, no. 9, pp. 614-621, 2005.

[69] L. A. Pinczewski, J. Lyman, L. J. Salmon, V. J. Russell, J. Roe, and J. Linklater, "A 10-year comparison of anterior cruciate ligament reconstructions with hamstring tendon and patellar tendon autograft: a controlled, prospective trial," The American Journal of Sports Medicine, vol. 35, no. 4, pp. 564-574, 2007.

[70] M. A. Risberg, I. Holm, H. Steen, and B. D. Beynnon, "Sensitivity to changes over time for the IKDC form, the Lysholm score, and the Cincinnati knee score. A prospective study of 120 ACL reconstructed patients with a 2-year follow-up," Knee Surgery, Sports Traumatology, Arthroscopy, vol. 7, no. 3, pp. 152-159, 1999.

[71] J. J. Irrgang, A. F. Anderson, A. L. Boland et al., "Development and validation of the international knee documentation committee subjective knee form," The American Journal of Sports Medicine, vol. 29, no. 5, pp. 600-613, 2001.

[72] J. Hoher, T. Bach, A. Münster, B. Bouillon, and T. Tiling, "Does the mode of data collection change results in a subjective knee score? Self-administration versus interview," The American Journal of Sports Medicine, vol. 25, no. 5, pp. 642-647, 1997.

[73] E. C. Rodriguez-Merchan, "Knee instruments and rating scales designed to measure outcomes," Journal of Orthopaedics and Traumatology, vol. 13, no. 1, pp. 1-6, 2012.

[74] E. M. Roos and S. Toksvig-Larsen, "Knee injury and Osteoarthritis Outcome Score (KOOS) - validation and comparison to the WOMAC in total knee replacement," Health and Quality of Life Outcomes, vol. 1, article 17, 2003.
[75] A. Almangoush, L. Herrington, I. Attia et al., "Cross-cultural adaptation, reliability, internal consistency and validation of the Arabic version of the Knee injury and Osteoarthritis Outcome Score (KOOS) for Egyptian people with knee injuries," Osteoarthritis and Cartilage, vol. 21, no. 12, pp. 1855-1864, 2013.

[76] S. Polgar and S. A. Thomas, Introduction to Research in the Health Sciences, Churchill Livingstone, London, UK, 4th edition, 2000. 


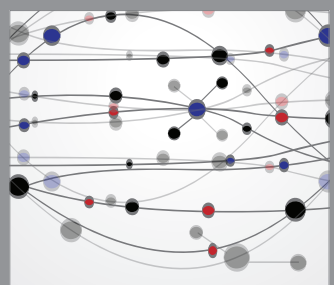

The Scientific World Journal
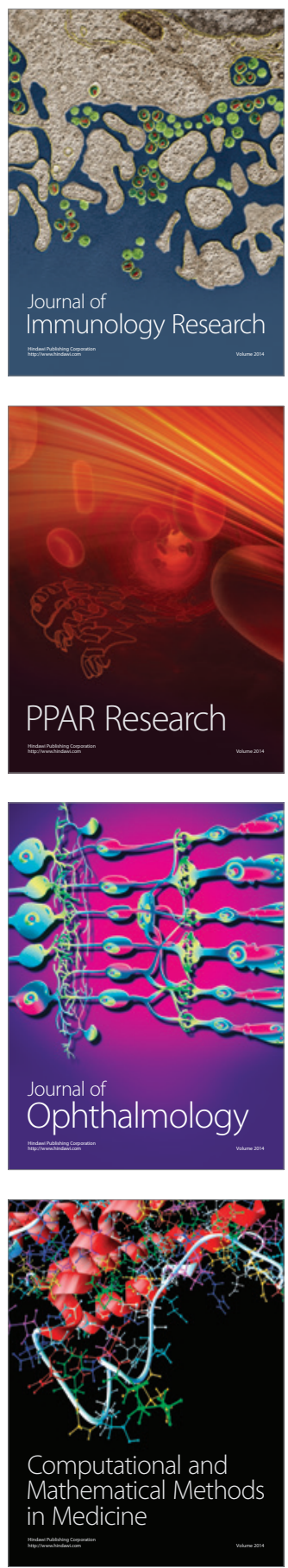

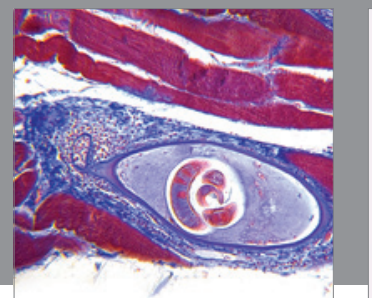

Gastroenterology

Research and Practice
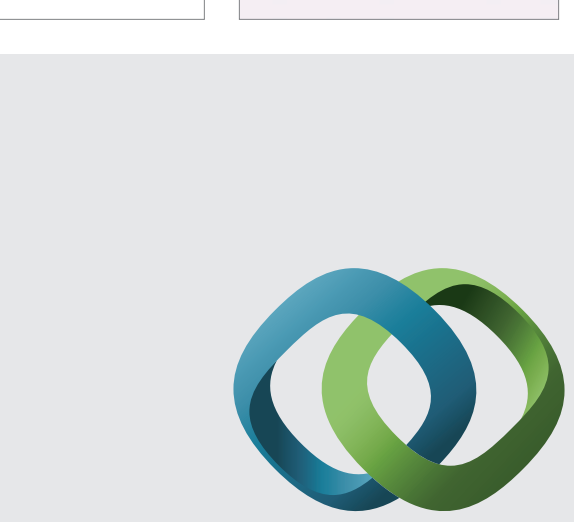

\section{Hindawi}

Submit your manuscripts at

http://www.hindawi.com
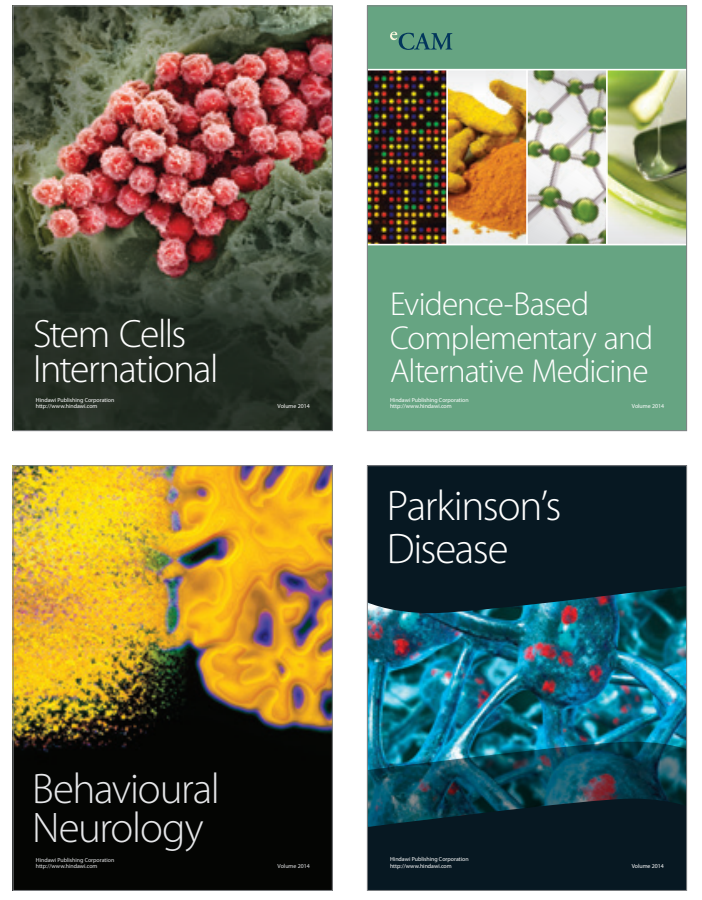
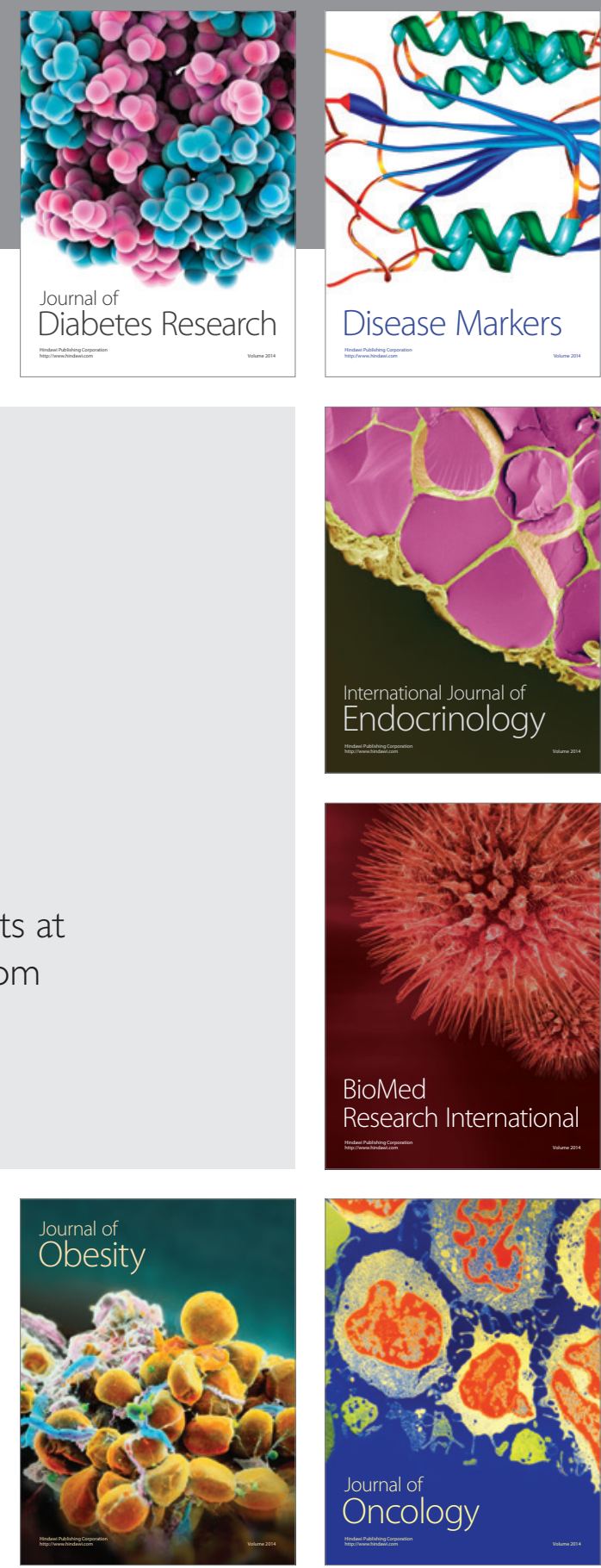

Disease Markers
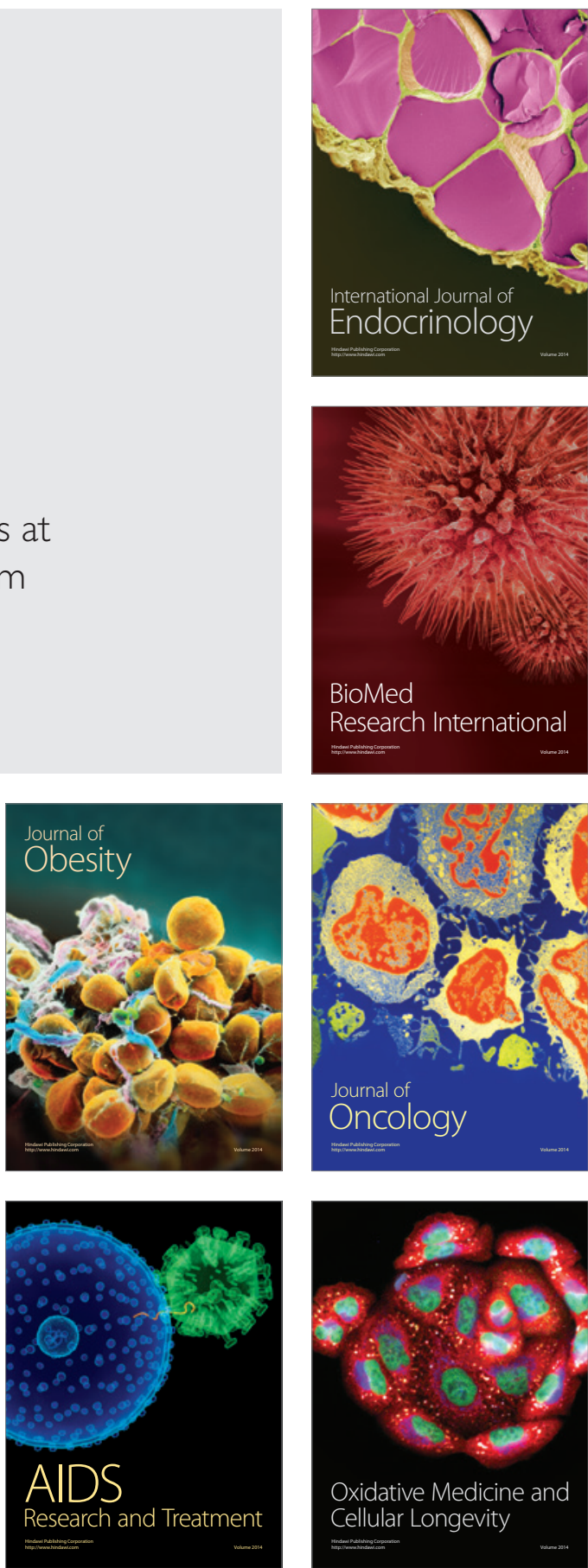